\title{
Novel approaches to pulmonary fibrosis
}

\author{
Authors: Gisli Jenkins ${ }^{\mathrm{A}}$ and Amanda Goodwin ${ }^{\mathrm{B}}$
}

\begin{abstract}
Idiopathic pulmonary fibrosis (IPF) is a devastating condition with a poor prognosis and few treatment options. However, recent research into this condition has led to considerable insights into the pathophysiology of the disease, resulting in the identification of potential biomarkers to aid diagnosis and stratification of patients and the development of novel therapies. In this review we will discuss the recent developments in this field and review how this knowledge has been translated into clinical trials and a paradigm shift in our approach to patients with IPF.
\end{abstract}

KEYWORDS: Idiopathic pulmonary fibrosis, biomarkers, pirfenidone, nintedanib

\section{Introduction}

Idiopathic pulmonary fibrosis (IPF) is a progressive lung disease of uncertain aetiology with limited therapeutic options and a dismal prognosis. There are around 5,000 new cases of IPF each year in the UK, and the incidence is climbing. ${ }^{1}$ IPF has a median survival of just 3 years and an estimated 5-year survival rate of $37 \%,{ }^{1}$ worse than many cancers.

The significant and increasing disease burden associated with IPF warrants considerable research to improve our understanding of the disease. However, until relatively recently research into IPF has been neglected compared with other conditions, such as cancer. Recent discoveries around the underlying pathophysiology of IPF have stimulated the development of novel therapies and new methods for diagnosis and assessment of prognosis. This review will discuss these developments.

\section{Diagnosis of IPF}

IPF typically presents in older patients with progressive dyspnoea and cough, and on examination bibasal inspiratory crackles and finger clubbing can be found. ${ }^{2}$ The natural history of IPF varies considerably. Many patients experience a gradual decline in respiratory function, however, some people have stable disease, whereas others experience very rapid deterioration in their condition. Acute exacerbations of IPF,

Authors: Areader in pulmonary biology and honorary consultant physician, Centre for Respiratory Research, University of Nottingham, Nottingham, UK; ${ }^{\mathrm{B}} \mathrm{CT} 2$ medicine, Nottingham University Hospitals NHS Trust, Nottingham, UK associated with sudden declines in clinical condition following a period of stability, affect a significant subgroup of patients. ${ }^{2}$ Importantly, the fibrotic changes of IPF are irreversible, and patients eventually succumb to respiratory failure.

International guidelines for the diagnosis of IPF were published in 2011 and are based on a combination of histological and high-resolution computed tomography (HRCT) findings (Fig 1). Crucially, if HRCT does not reveal typical usual interstitial pneumonia features, a surgical lung biopsy is recommended to confirm the diagnosis. ${ }^{2}$ However, many patients are not fit enough to undergo this high-risk procedure, and so may lack a formal diagnosis of IPF. Patients with pulmonary fibrosis of unspecified origin make up the second largest sub-group of many interstitial lung disease (ILD) registries but they may not receive adequate support in terms of access to new therapies or clinical trials. Therefore, novel imaging strategies that can identify molecular mechanisms of IPF may improve current diagnostic methods. ${ }^{3}$

\section{Pathogenesis of IPF}

The molecular mechanisms of IPF are incompletely understood, but alveolar epithelial cells and fibroblasts are thought to play key roles. The current pathophysiological paradigm suggests an abnormal wound healing response of the alveolar epithelium. Sources of the initiating injury include cigarette smoke, industrial dusts, gastro-oesophageal reflux and viral infection, which in susceptible individuals, activate inflammatory cascades and increased expression of pro-fibrotic cytokines. ${ }^{4}$ For fibrogenesis to occur there must be failure of alveolar epithelial repair, via increased apoptosis and premature senescence of epithelial cells. ${ }^{5}$ This results in increased myofibroblasts with enhanced extracellular matrix deposition within the pulmonary interstitium, leading to the characteristic lesions of IPF known as fibroblastic foci. The consequent distortion of the pulmonary structure impairs gas exchange, resulting in respiratory failure ${ }^{4}$ (Fig 2).

An area of particular interest in IPF is the role of transforming growth factor $\beta$ (TGF $\beta$ ). TGF $\beta$ is a pro-fibrotic cytokine that is crucial to many processes involved in IPF, including epithelial cell migration, epithelial apoptosis, fibroblast proliferation, transformation of fibroblasts into myofibroblasts, and collagen synthesis. ${ }^{6}$ TGF $\beta$ must be activated in order to exert any profibrotic effects, which can occur via several processes, including extremes of $\mathrm{pH}$ or temperature and proteases. However, the best characterised mechanism of TGF $\beta$ activation in IPF is integrin-mediated. ${ }^{6}$ 

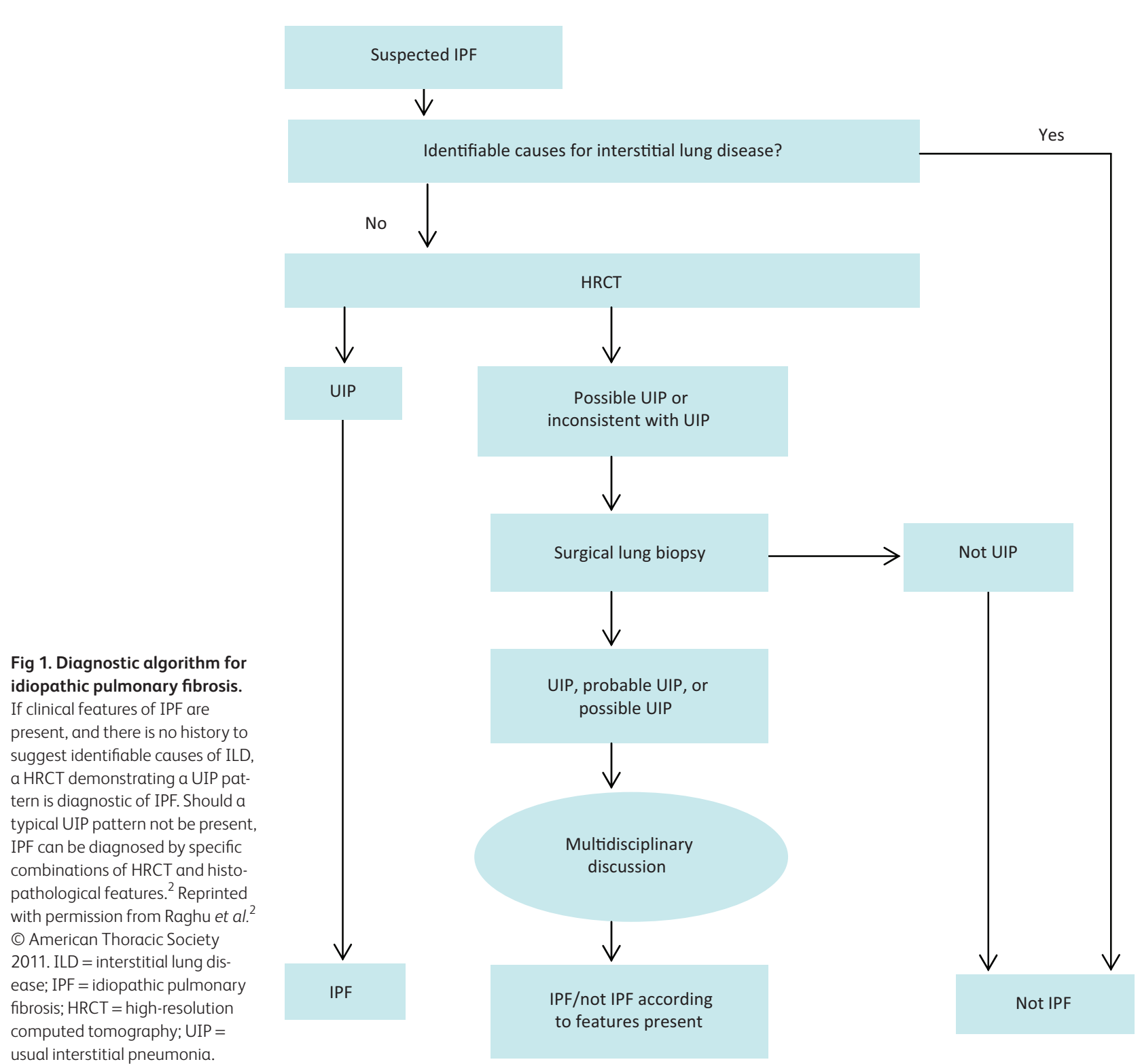

usual interstitial pneumonia.

Integrins are heterodimeric cell membrane proteins that consist of $\alpha$ and $\beta$ subunits. Several integrins activate TGF $\beta$, including $\alpha v \beta 1, \alpha v \beta 3, \alpha v \beta 5, \alpha v \beta 6$ and $\alpha v \beta 8{ }^{6,7} \alpha v \beta 6$ mediated TGF $\beta$ activation is best characterised in IPF. ${ }^{6,8,9}$ However, loss of the $\alpha \mathrm{v}$ gene is protective in animal models of organ fibrosis, emphasising the importance of the $\alpha \mathrm{v}$ integrins in fibrotic disease and highlighting them as potential therapeutic targets. ${ }^{10}$

The majority of IPF is sporadic, but up to $20 \%$ of cases are familial. ${ }^{11}$ Genetic studies have implicated genes involved in host defence, inter-cellular adhesion and DNA repair in the pathogenesis of IPF. ${ }^{12}$ Abnormalities of telomerase and surfactant protein $\mathrm{C}$ and $\mathrm{A} 2$ have also been described. ${ }^{4}$ Further evaluation of familial cases of IPF may identify key pathological processes involved in the development of IPF.

Further research to dissect the molecular pathways involved in IPF is essential. Work should aim to establish which pathways cause fibrosis, and which are driven by the fibrotic process, thus identifying potential therapeutic targets.

\section{IPF - a malignant disease?}

Several of the hallmarks of cancer have been described in IPF, leading some to argue that IPF should be considered a malignant disease. Cancer and IPF are both fatal conditions that involve aberrant cellular proliferation. In addition, mutations in tumour suppressor genes have been found in tissue samples from patients with IPF. ${ }^{13}$ Furthermore, the differentiation and infiltration of myofibroblasts seen in IPF has been likened to the tissue invasion that is typical of malignancy. ${ }^{13}$ However, IPF is always bilateral, does not cause extra-pulmonary metastases, and fibroblasts within fibroblastic foci do not originate from a single clone, ${ }^{13}$ all of which argue against IPF being a malignant disease. 
Fig 2. The molecular mechanisms of idiopathic pulmonary fibrosis. 1) Injurious agents, such as cigarette smoke, industrial dusts, gastro-oesophageal reflux and viral infections, cause alveolar epithelial injury. 2) Inflammatory and pro-fibrotic processes are initiated in susceptible individuals. 3) Alveolar epithelial repair fails secondary to increased apoptosis and premature senescence. 4) Formation of fibroblastic foci, resulting in distortion of the pulmonary structure and impaired gas exchange. Adapted with permission from Goodwin and Jenkins. ${ }^{6}$ (c) the Biochemical Society 2009.

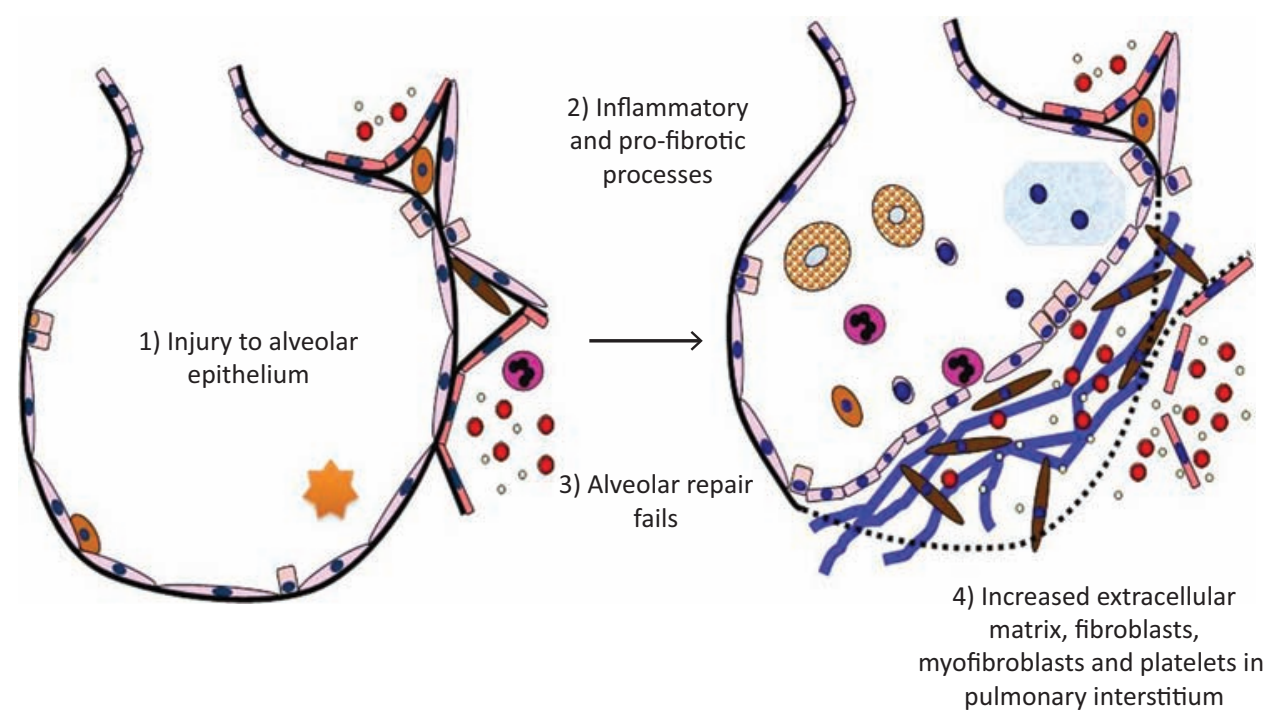

Although IPF is not a primary malignant condition, there could be benefits to comparing IPF with cancer. For example, our knowledge of cancer biology has increased dramatically in recent decades, leading to the development of prognostic markers, new therapies, individualised treatment strategies and improved survival. ${ }^{14}$ Using similar approaches to those adopted in oncology, both in terms of research and cancer pathways, could lead to the dramatic improvements that have been observed in oncology over the last 40 years. Furthermore, both cancer and IPF share abnormal cellular signalling pathways, and existing cancer treatments may be beneficial in IPF. ${ }^{13}$ While theoretically many of the principles used in oncology could be applied to IPF, further studies are required to confirm this.

\section{Biomarkers in IPF}

The clinical management of IPF is rife with problems due to difficulties in the diagnosis, prognostication and management of the condition. The current guidelines recommend that patients with a poorer prognosis should be considered early for lung transplantation, ${ }^{2}$ but accurate methods of assessing prognosis are currently lacking. In addition, non-invasive diagnostic tests are required to replace surgical lung biopsy. Finally, although there are now a few therapeutic options available, the disease is ultimately fatal and individualised treatment strategies have not been adopted in IPF. These issues have prompted the search for reliable and easy-to-measure markers to aid diagnosis, assess prognosis and guide individualised treatment in IPF.

A polymorphism in the promoter region of the mucin gene MUC5B has been linked with a higher risk of developing IPF, but has also been associated with improved survival if present. ${ }^{15,16}$ The relationship of this common polymorphism with the risk of disease and survival suggests that it could be used as a diagnostic and prognostic marker.

In addition, multiple serum biomarkers have been found to predict poor outcomes in IPF. These include markers of alveolar cell epithelial injury, mucin-1 (also known as KL-6), surfactant protein-A (SP-A) and matrix metallopreotease-7 (MMP-7), the marker of alveolar macrophage activation chemokine ligand-18 (CCL-18), markers of neutrophil recruitment S100A12 and interleukin-8 (IL-8), and indicators of oxidative stress intercellular adhesion molecule-1 (ICAM-1) and vascular cell adhesion molecule-1 (VCAM-1) ${ }^{17}$ In Japan, KL- 6 and the SP-A and $-\mathrm{D}$ are used as diagnostic markers for ILD, but these cannot accurately distinguish between IPF and other forms of ILD. ${ }^{18}$

The existing studies of biomarkers in IPF have involved small patient populations; therefore, no biomarker is validated for diagnosis or prognosis at the current time. The on-going study PROFILE (Prospective Observation of Fibrosis in the Lung Clinical Endpoints) aims to validate some of these biomarkers in newly diagnosed patients (clinicaltrials.gov reference NCT01134822). ${ }^{19}$

The identification of validated biomarkers in IPF will allow earlier detection, a more predictable prognosis, and personalised treatment strategies. Ongoing research into the pathogenesis of IPF is required to identify groups of patients that are more likely to benefit from new therapies. In addition, accurate prognostic markers will indicate which patients should be prioritised for lung transplantation.

\section{Clinical trials in IPF}

Until recently, the only therapy to improve survival in IPF was lung transplantation. This is not a feasible option for many patients with IPF due to a number of contra-indications, thus interest has switched to pharmacological management. Many clinical trials have yielded negative results for pharmacological agents ${ }^{20}$ and emerging evidence has cast doubt upon treatment regimens previously considered to be standard therapies.

\section{$\mathrm{N}$-acetylcysteine and immunosuppression for IPF}

It was previously thought that inflammation was a key event in a pathogenesis of IPF, therefore it was postulated that immunosuppressive therapy could slow progression of the disease. The immunosuppressive combination of azathioprine and prednisolone was considered to be a standard treatment for IPF, but there was limited evidence for its use.

Another therapy widely used in IPF is $\mathrm{N}$-acetylcysteine (NAC). NAC increases pulmonary glutathione levels, and is 
postulated to restore the oxidant-antioxidant imbalance that may be contributing to the disease process of IPF. ${ }^{21}$ Both NAC and immunosuppression have been assessed in recent clinical trials to clarify their roles in the management of IPF.

The IFIGENIA study found that the addition of NAC to standard therapy (azathioprine and prednisolone) significantly slowed deterioration in lung function compared with placebo. ${ }^{21}$ On the basis of these findings, triple therapy with NAC, prednisolone and azathioprine was commenced in many patients with IPF. Unfortunately, more recent evidence suggests that NAC and immunosuppressive therapy have no benefit in IPF, and could potentially be harmful. The PANTHER study assessed the combination of NAC, azathioprine and prednisolone against NAC or placebo alone, but was terminated prematurely when an interim analysis demonstrated that triple therapy increased the risk of death and hospitalisation. ${ }^{22}$ The NAC and placebo arms of this trial continued, but found no difference in pulmonary function, death rates or acute exacerbations between NAC and placebo in patients with mild fibrosis. ${ }^{23}$

While NAC may still be of benefit to patients with more advanced IPF who were not included in the above studies, it is clear that the focus should now be to trial new agents in IPF.

\section{Pirfenidone - the first specific anti-fibrotic therapy}

Pirfenidone was the first drug to be licensed specifically for IPF. Pirfenidone exerts anti-fibrotic, anti-inflammatory and antioxidant effects, via inhibition of multiple pathways implicated in the pathogenesis of IPF. ${ }^{24}$ Four phase 3 studies of pirfenidone in IPF have been published.

The first three trials gave varying results. A Japanese study found that pirfenidone reduced the decline in vital capacity compared with placebo at one year. ${ }^{24}$ The CAPACITY-2 trial also found that pirfenidone significantly reduced the deterioration in forced vital capacity (FVC) compared with placebo at 72 weeks, but the concurrent CAPACITY-1 study did not replicate these results. ${ }^{25}$ A Cochrane meta-analysis of these three trials showed that pirfenidone significantly reduced the risk of disease progression $(30 \%$ reduction, hazard ratio 0.70 , $95 \%$ CI $0.56-0.88, p=0.002)$, defined as death and/or a $10 \%$ or more decline in vital capacity from baseline. ${ }^{26}$

The fourth phase 3 trial of pirfenidone in IPF, the ASCEND trial, was designed to clarify the results of the earlier studies.
Pirfenidone significantly improved progression-free survival (hazard ratio $0.57,95 \%$ CI $0.43-0.77, \mathrm{p}<0.001$ ) and slowed the decline in FVC (relative difference in decline between groups $45.1 \%, \mathrm{p}<0.001)$ at 52 weeks, ${ }^{27}$ confirming the positive results seen in earlier studies. Moreover, this study reported promising survival statistics.

Before pirfenidone, no pharmacological therapies improved survival in IPF. The CAPACITY trials reported a trend towards improved mortality with pirfenidone, ${ }^{25}$ but this was not statistically significant. A pre-defined pooled analysis of patients from the ASCEND and CAPACITY trials revealed a reduction in all-cause mortality with pirfenidone compared with placebo. ${ }^{27}$

Importantly, these trial findings can only be applied to the populations studied (generally those with mild-to-moderate disease). Subgroup analysis of the Japanese trial showed that pirfenidone was most effective in patients with early IPF. ${ }^{28}$ At present, pirfenidone is approved by the National Institute for Health and Care Excellence (NICE) only for patients with FVC measurements of $50-80 \%$ predicted. ${ }^{29}$ Further research is required to assess the role of pirfenidone in more advanced disease.

\section{Nintedanib - an anti-cancer drug slows progression of IPF}

Nintedanib is a small molecule inhibitor that was initially developed as an anticancer agent. Nintedanib inhibits multiple tyrosine kinases implicated in fibrogenesis, including the receptors for vascular endothelial growth factor, fibroblast growth factor and platelet-derived growth factor. ${ }^{30}$

In May 2014, the results of the concurrent phase 3 trials of nintedanib versus placebo, INPULSIS-1 and INPULSIS-2, were reported. Nintedanib significantly reduced the decline in FVC compared with placebo in INPULSIS-1 (difference of $125.3 \mathrm{ml}, 95 \%$ CI $77.7-172.8 \mathrm{ml}, \mathrm{p}<0.001)$ and INPULSIS-2 (difference of $93.7 \mathrm{ml}, 5 \% \mathrm{CI} 44.8-142.7 \mathrm{ml} \mathrm{p}<0.001$ ) after one year. ${ }^{30}$ There was also a trend towards reduced death rate with nintedanib, but the study was not powered to detect differences in mortality. The results indicated that nintedanib is a well-tolerated drug that may slow disease progression in IPF, thus making it the first anti-cancer agent to show benefit in IPF. NICE have yet to issue guidance on the use of nintedanib

Table 1. Ongoing clinical trials of novel agents in IPF.

\begin{tabular}{|c|c|c|c|}
\hline Agent & Trial & Mechanism of action & Estimated completion date \\
\hline $\begin{array}{l}\text { Tralokinumab MedImmune/ } \\
\text { AstraZeneca }\end{array}$ & $\begin{array}{l}\text { NCT01629667, } \\
\text { NCT02036580 }\end{array}$ & Anti-IL-13 monoclonal antibody & November 2015-January 2016 \\
\hline FG 3019 FibroGen & NCT01890265 & Anti-connective tissue growth factor antibody & July 2016 \\
\hline $\begin{array}{l}\text { BMS-986020 Bristol-Myers } \\
\text { Squibb }\end{array}$ & NCT01766817 & Lysophosphatidic acid receptor antagonist & April 2016 \\
\hline Lebrikuzumab Roche & NCT01872689 & Anti-IL-13 monoclonal antibody & August 2016 \\
\hline Simtuzumab Gilead & NCT01769196 & Lysyl oxidase homolog 2 inhibitor & February 2018 \\
\hline SAR156597 Sanofi-Aventis & NCT01529853 & Anti-IL-4/IL-13 monoclonal antibody & Completed October 2013 \\
\hline STX-100 Stromedix & NCT01371305 & Anti- $\alpha \vee \beta 6$ integrin antibody & Completed December 2013 \\
\hline
\end{tabular}


in IPF, but this drug is likely to play a key role in the future management of this disease.

\section{Future developments}

Recent investigations into the pathogenesis of IPF have revealed potential targets for novel therapies and several new agents are currently being investigated for use in IPF (Table 1). It is hoped that these studies may reveal new agents to join pirfenidone and nintedanib in improving outcomes for patients with IPF.

The heterogeneity of IPF suggests that a single therapeutic agent will not be effective for all. Individualised treatment plans, similar to those used in oncology, may maximise desired outcomes while minimising cost and adverse effects. Future clinical trials should aim to identify subgroups of patients according to their response to experimental drugs, as well as assessing novel targeted agents.

Future study should also focus on new diagnostic and prognostic strategies. The pathogenesis of IPF is multifactorial and involves complex pathological mechanisms. Therefore, it is unlikely that a single disease marker will be sufficient to accurately diagnose and indicate the prognosis of IPF. Scoring systems that integrate validated physiological, radiological and biomarker assessment should be developed in future to aid the diagnosis and assessment of prognosis of IPF.

\section{Conclusion}

IPF is a progressive disease with a dismal prognosis. Advances in our understanding of the mechanisms of IPF have revealed novel methods for the diagnosis and stratification of patients, as well as molecular therapeutic targets. Standard pharmacological therapies have proven disappointing in clinical trials, but two new agents have recently shown promising results. Further evaluation of these agents and other emerging therapies is required to improve outcomes for patients with IPF.

\section{References}

1 Navaratnam V, Fleming KM, West J et al. The rising incidence of idiopathic pulmonary fibrosis in the UK. Thorax 2011;66:462-7.

2 Raghu G, Collard HR, Egan JJ et al. An official ATS/ERS/JRS/ALAT statement: Idiopathic pulmonary fibrosis: Evidence-based guidelines for diagnosis and management. Am J Respir Crit Care Med 2011;183:788-824.

3 John AE, Luckett JC, Tatler AL et al. Preclinical SPECT/CT imaging of $\alpha v \beta 6$ integrins for molecular stratification of idiopathic pulmonary fibrosis. J Nucl Med 2013;54:2146-52.

4 Camelo A, Dunmore R, Sleeman MA, Clarke DL. The epithelium in idiopathic pulmonary fibrosis: breaking the barrier. Front Pharmacol 2014;4:173.

5 Chilosi M, Carloni A, Rossi A et al. Premature lung aging and cellular senescence in the pathogenesis of idiopathic pulmonary fibrosis and COPD/ emphysema. Transl Res 2013;162:156-73.

6 Goodwin A, Jenkins G. Role of integrin-mediated TGF $\beta$ activation in the pathogenesis of pulmonary fibrosis. Biochem Soc Trans 2009;7:849-54.

7 Tatler AL, John AE, Jolly L et al. Integrin $\alpha \mathrm{v} \beta 5$ mediated TGF- $\beta$ activation by airway smooth muscle cells in asthma. J Immunol 2011;187:6094-107.

8 Jenkins RG, Su X, Su G et al. Ligation of protease-activated receptor 1 enhances $\alpha v \beta 6$ integrin-dependent TGF- $\beta$ activation and promotes acute lung injury. J Clin Invest 2006;116:1606-14.
9 Xu MY, Porte J, Knox AJ et al. Lysophosphatidic acid induces $\alpha v \beta 6$ integrin-mediated TGF- $\beta$ activation via the LPA2 receptor and the small $\mathrm{G}$ protein $\mathrm{G} \alpha_{\mathrm{q}}$. Am J Pathol 2009;174:1264-79.

10 Henderson NC, Arnold TD, Katamura Y et al. Targeting of $\alpha \mathrm{v}$ integrin identifies a core molecular pathway that regulates fibrosis in several organs. Nat Med 2013;19:1617-24.

11 Wuyts WA, Agnostini C, Antoniou K et al. The pathogenesis of pulmonary fibrosis: a moving target. Eur Respir J 2013;41:1207-18.

12 Fingerlin TE, Murphy E, Zhang W et al. Genome-wide association study identified multiple susceptibility loci for pulmonary fibrosis. Nat Genet 2013;45:613-20.

13 Vancheri C. Common pathways in idiopathic pulmonary fibrosis and cancer. Eur Respir Rev 2013;22:265-72.

14 Cancer Research UK. Cancer Statistics Report: Cancer Mortality in the UK in 2011. London: CRUK, 2013.

15 Seibold MA, Wise AL, Speer MC et al. A common MUC5B promoter polymorphism and pulmonary fibrosis. $N$ Eng J Med 2011;364:1503-12.

16 Peljto AL, Zhang Y, Fingerlin TE et al. Association between the MUC5B promoter polymorphism and survival in patients with idiopathic pulmonary fibrosis. JAMA 2013;309:2232-2239.

17 Richards TJ, Kaminski N, Baribaud et al. Peripheral blood proteins predict mortality in idiopathic pulmonary fibrosis. Am I Respir Crit Care Med 2012;185:67-76.

18 Huang H, Peng X, Nakajima J. Advances in the study of biomarkers of idiopathic pulmonary fibrosis in Japan. Biosci Trends 2013;7:172-7.

19 Maher TM. PROFILEing idiopathic pulmonary fibrosis: rethinking biomarker discovery. Eur Respir Rev 2013;128:148-52.

20 Woodcock HV, Maher TM. The treatment of idiopathic pulmonary fibrosis. F1000 Prime Rep 2014;6:16.

21 Demedts M, Behr J, Buhl R et al. High-dose acetylcysteine in idiopathic pulmonary fibrosis. N Eng J Med 2005;353:2229-42.

22 The Idiopathic Pulmonary Fibrosis Clinical Research Network. Prednisolone, azathioprine, and $\mathrm{N}$-acetylcysteine for pulmonary fibrosis. N Eng J Med 2012;366:1968-77.

23 The Idiopathic Pulmonary Fibrosis Clinical Research Network. Randomized trial of acetylcysteine in idiopathic pulmonary fibrosis. N Eng J Med 2014;370:2093-101.

24 Taniguchi H, Ebina M, Kondoh Y et al. Pirfenidone in idiopathic pulmonary fibrosis. Eur Respir J 2010;35:821-9.

25 Noble PW, Albera C, Bradford WZ et al. Pirfenidone in patients with idiopathic pulmonary fibrosis (CAPACITY): two randomised trials. Lancet 2011;377:1760-9.

26 Spagnolo P, Del Giovane C, Luppi F et al. Non-steroid agents for idiopathic pulmonary fibrosis (review). Cochrane Database Syst Rev 2010;Sept 8:CD003134.

27 King TE, Bradford WZ, Castro-Bernardini S et al. A phase 3 trial of pirfenidone in patients with idiopathic pulmonary fibrosis. $\mathrm{N} \mathrm{Eng} \mathrm{J}$ Med 2014;370:2083-92.

28 Azuma A, Taguchi Y, Ogura T et al. Exploratory analysis of a phase III trial of pirfenidone identifies a subpopulation of patients with idiopathic pulmonary fibrosis as benefiting from treatment. Respir Res 2011;12:143.

29 National Institute for Health and Clinical Evidence. Pirfenidone for treating idiopathic pulmonary fibrosis (NICE technology appraisal guidance). NICE, 2013. Available online at http://guidance.nice.org. $\mathrm{uk} / \mathrm{ta2} 22$ [Accessed 2 June 2014].

30 Richeldi L, du Bois RM, Raghu G et al. Efficacy and safety of nintedanib in idiopathic pulmonary fibrosis. $N$ Engl J Med 2014;370:2071-82.

Address for correspondence: $\operatorname{Dr} \mathrm{G}$ Jenkins, Centre for Respiratory Research, Clinical Sciences Building, City Campus, Hucknall Road, Nottingham, NG5 1PB, UK.

Email: gisli.jenkins@nottingham.ac.uk 